\title{
Immunotherapy in tumors failing check-point inhibitors: the sarcoma example - What we missed and where we are heading
}

\author{
Alessandra Merlini ${ }^{1,2}$, Francesco Tolomeo ${ }^{1,2}$, Sara Miano ${ }^{1,2}$, Lorenzo D’Ambrosio ${ }^{1,2}$, \\ Dario Sangiolo ${ }^{1,2}$, Giovanni Grignani ${ }^{1}$
}

\begin{abstract}
The introduction of immune checkpoint inhibitors represented a true revolution in the treatment of melanoma and a few other cancer subtypes. Unfortunately, the use of these drugs did not achieve the same beneficial results in other neoplasms, such as soft tissue sarcoma and gastrointestinal stromal tumor. These failures encouraged deeper research into the complex interactions between cancer and host immune system, to try to shed light on the ability of cancer cells to escape immunologic surveillance. Key elements to explain tumor immune escape were found in the tumor microenvironment. The main actors in this complex network are lymphocytes, cytokines and innate immunity cells such as macrophages and antigen presenting cells. Thus, immuno-oncologists are studying the different components of the tumor microenvironment to identify possible new therapeutic targets. In this paper, we summarize the most important aspects of these interactions, and provide an overview of the newer and more promising immunotherapeutic strategies.
\end{abstract}

Key words: immunotherapy, checkpoint inhibitor, immune escape, tumor microenvironment

\section{Introduction}

The first attempt to use immune system (IS) against tumors dates back to more than a century ago. In 1890 William Coley, a surgeon of New York City, directly injected streptococcal bacteria into inoperable tumors with notable successes in regression of tumors [1]. However, in the following decades, cancer immunotherapy was considered to be ineffective and irreproducible. Since the early 1990s, researchers have developed several ways to stimulate the patient's IS, some of which have been successful and some less so, depending on the type of cancer [2]. The introduction of checkpoint inhibitors targeting cytotoxic T/lymphocyte-associated protein $4(\mathrm{CTLA} / 4)$ or programmed cell-death protein 1

\footnotetext{
'Sarcoma Unit, Medical Oncology, Candiolo Cancer Institute FPO IRCCS, Candiolo, Torino, Italy.

2Department of Oncology, University of Torino Medical School, Candiolo, Torino, Italy.

Correspondence to:

Giovanni Grignani, MD,

Medical Oncology, Candiolo Cancer Institute FPO, IRCCS,

Strada Provinciale 142, Km 3.95, 10060 Candiolo (Torino), Italy.

Phone: +390119933623 - Fax: +390119933290

E-mail: giovanni.grignani@ircc.it

CANCER BREAKING NEWS 2017;5(2):22-32

DOI: $10.19156 / \mathrm{cbn} .2017 .0045$
}

(PD-1), or programmed cell-death 1 ligand 1 (PD-L1), depending on the drug considered, was undoubtedly a revolution in cancer treatment. These molecules have demonstrated clinical activity in more than 15 different cancer types, including melanoma, non-small-cell lung carcinoma (NSCLC), renal cell carcinoma (RCC), bladder carcinoma and Hodgkin lymphoma [3]. Despite initial enthusiasm, the use of these drugs did not achieve the same results in other tumors. For example, in gastrointestinal stromal tumor (GIST) the use of ipilimumab in combination with dasatinib did not show any significant activity, while ipilimumab in patients with recurrent synovial sarcoma failed to show relevant clinical activity [4].

It is now clear that the "melanoma miracle" will not be reproducible in the great majority of other cancer histotypes. These failures prompted immuno-oncologists to investigate more deeply into several different aspects of the cancer host immune-response, coming to the conclusion that is an element of uniqueness in every tumor histotype and patient. Only the knowledge of this uniqueness will allow the expansion of the possibilities of immunotherapy to a broader number of patients. Indeed, several layers of complexity need to be understood to elucidate the interactions between tumor and the IS. In 
this paper, we will describe some key features of the IStumor interaction, with a specific focus on the tumor microenvironment (TME) in order to identify new potential targets and design new immunotherapeutic strategies.

\section{Tumor and host: a complex relationship}

\section{Lymphocytic infiltrate}

Teng and collaborators proposed an elegant classification of TMEs [5], based on previous findings in melanoma [6]. The selected variables are tumor-infiltrating lymphocytes (TIL; presence or absence) and PD-L1 positivity, generating four possible scenarios: Type I: PD-L1 positive, TILs present (adaptive immune resistance); Type II: PD-L1 negative, no TILs (immune ignorance); Type III: PD-L1 positive, no TILs (intrinsic induction); Type IV: PD-L1 negative with TILs, implying that other pathways could be implicated in immune tolerance. The temporal and spatial intrinsic variability of PD-L1 expression, i.e., its dynamic expression at different time points in tumor progression and its significance depending on the cell type expressing PD-L1, effectively hampers the application of this stratification. Furthermore, important actors of host-tumor interplay, such as macrophages and myeloid-derived suppressor cells (MDSC), are not taken into account in this classification.

Beyond the prognostic role of TILs in almost all neoplasms, the crucial role played by lymphocyte infiltration in immune checkpoint inhibitor therapy was elegantly shown by Tumeh and collaborators [7]. These authors formally demonstrated that in the absence of TILs no response can be achieved with immune checkpoint inhibitors alone.

Within the different tumor types in which immune checkpoint inhibitors were not able to achieve clinically relevant results, soft tissue sarcomas (STS) have some peculiarities. Indeed, STS are a rare and extremely heterogeneous group of tumors encompassing more than fifty different histotypes $[8,9]$. This heterogeneity is both a challenge and an intriguing model for the study of finetuning mechanisms of the TME. For instance, in a recent paper [10] the authors compared the mutational burden of different STS (undifferentiated pleomorphic sarcoma [UPS], leiomyosarcoma [LMS], well-differentiated/dedifferentiated liposarcoma [WD/DD LPS], synovial sarcoma [SS] and myxoid-round cell liposarcoma [MRCL]) and their different lymphocytic infiltrate. The authors found a positive correlation between mutational burden, immunogenicity, an abundance of oligoclonal T-cell infiltrates and the great variability of the microenvironmental immune asset among histotypes. As an example,
LMS is both largely immunogenic and rich in immunosuppressive tumor-associated macrophages, suggesting that targeting both adaptive immunity through immune checkpoints and innate immunity might provide better responses. Indeed, the anti PD-1 inhibitor nivolumab alone was not able to show any relevant activity in uterine LMS [11]. Taking inspiration from this example, it is clear that having effective biomarkers to evaluate, follow and measure the variations in TME, and consequently in tumor response to immunotherapy, would be of utmost importance.

To date, the most widely accepted parameters to evaluate tumor immunogenicity, and possibly predict response, are: PD-L1 expression (with all the well-known caveats concerning the determination of expression), percentage of CD8+ T cells, T cell clonality, presence of regulatory T-cells $\left(\mathrm{T}_{\text {regs }}\right)$ and macrophagic infiltrate.

\section{Cytokines}

A relevant predictor of response to immunotherapy is the abundance in the TME of MDSC. These cells are true "cytokine factories" and are key players at the frontline of immune suppression. The cytokine family of molecules is composed of soluble proteins, mainly but not exclusively secreted by cells of the IS, with diverse roles across physiology and pathology. Differently from the well-known, almost uniquely endocrine function of hormones, cytokines can act in a paracrine manner, exerting their action on neighbor cells, or directly affecting the producer cells in an autocrine fashion. Cytokines are the "words" with which the IS communicates with other cells of the body. These molecules can act in synergy (different cytokines contribute to a common effect), quite often with redundant roles. Much like the meaning of words in a sentence depends on the semantic context, the same cytokine can be involved in several biological processes and even play seemingly opposite roles (pleiotropy). $\mathrm{Cy}-$ tokine crosstalk and trafficking are crucial at many steps in the metastatic process, such as epithelial-to-mesenchymal transitions (EMTs). The EMT is a key evolutionary conserved, developmental process which is hijacked early on by tumor cells in the invasion-metastasis cascade and which is almost entirely dependent on TME interactions [12]. One renowned player at many metastatic steps, including EMTs, is transforming growth factor- $\beta$ (TGF- $\beta$ ) [13]. TGF- $\beta$ signaling physiologically acts as a tumor suppressor, as it oversees differentiation, survival, and homeostasis of virtually all cell types in our body, but often changes side during carcinogenesis [14-16]. This cytokine is produced both by cancer parenchymal cells and by stromal cells such as myofibroblasts, and its 
associated pathways are involved both in tumor suppression and progression. This rather puzzling variability is related to the type of cancer and, even within the same tumor, to specific steps of tumor progression. There are studies suggesting that TGF- $\beta$ can act as a cytokine of metastasis in breast cancer and endometrial cancer, bladder cancer, melanomas and also sarcomas. Its role in sarcomas has been demonstrated as early as 1983, with the discovery that the "Sarcoma Growth Factor" was a mixture of two proteins, transforming growth factor- $\alpha$ (TGF- $\alpha$ ) and TGF- $\beta$ [17]. More recently, the gene encoding for TGF- $\beta$ type II receptor, harboring a tumorsuppressing function, has been identified as a major target of EWS-FLI1 [18].

Many cytokines and cytokine receptors have been under investigation as potential targets for combining agents targeting PD-L1/PD-1 and cytokines/chemokines with an immunosuppressive role $[19,20]$. We describe a few relevant examples.

Interleukin (IL)-10 is produced by T-helper (Th)2, Th1, Th17 (a subset of $\mathrm{T}_{\text {regs }}$ ), CD8 $+\mathrm{T}$ cells, B cells, and by cells of the innate IS such as macrophages, dendritic cells (DCs), natural killer (NK) cells, neutrophils and eosinophils, mast-cells. Its main targets are DCs and macrophages, contributing to the inhibition of antigen-presentation process $[21,22]$. IL-10 produced by macrophages in the TME can suppress IL-12 expression in DCs, thus impairing $\mathrm{CD} 8+\mathrm{T}$ cell-dependent response to chemotherapy [23]. Raised serum concentrations of IL-10 are associated with negative prognosis in at least 10 cancer types, among them bone sarcomas and lymphomas, possibly because of its potent immunosuppressive role at the TME-tumor interface.

Cytokine receptors (such as CSF1R). Colony-stimulating factor 1 (CSF1) is crucial for macrophage functioning and survival. CSF1 receptor (CSF1R) is expressed on the surface of tumor-associated macrophages (TAMs); its blockade leads to a reduction in TAM-promoting activity and decreased expression of M2 markers [24]. The combination of CSF1R inhibitors with immune checkpoint inhibitors is currently being investigated, among others, by the study exploring the association of PLX3397, an inhibitor of CSF1R (phase I/IIa study: A Combination Clinical Study of PLX3397 and Pembrolizumab To Treat Advanced Melanoma and Other Solid Tumors, https://clinicaltrials. gov/ct2/show/NCT02452424. Accessed July 15, 2017), with pembrolizumab, both in advanced melanoma and other solid cancers (NSCLC, ovarian cancer, squamous cell cancer of the head and neck, and GIST).

Finally, interferons (IFNs) are probably the most rele- vant class of cytokines to date in terms of our knowledge of their function and potential therapeutic applications. Indeed, IFN- $\boldsymbol{\gamma}$ is most likely the best example of the crosstalk and high level of complexity of the cytokine network. The main producers of this potent cytokine are antigen presenting cells (APCs), NK cells and T cells (both $\mathrm{CD} 4+$, in particular Th1, and CD8+). The main targets of IFN- $\gamma$ among immune cells are B lymphocytes, Th1 lymphocytes, and macrophages. Briefly, IFN- $\gamma$ induces human leukocyte antigen-D related (HLA-DR) expression and upregulates major histocompatibility molecules-class I (MHC-I) on most cells of the human body, especially in viral infections, for T-cell mediated recognition and killing. IL-10 negatively regulates IFN- $\gamma$ production, while TGF- $\beta$ indirectly impairs IFN- $\gamma$ production, by inhibiting CD4+ cells polarization by Th1, the main producers of IFN- $\gamma$. As an example, in Ewing sarcoma, IFN- $\gamma$ stimulates the production of chemokines (i.e., CXCL9 and CXCL10) by tumor and stromal cells. These cytokines are able to recruit $\mathrm{T}$ lymphocytes expressing the corresponding receptors, eventually leading to increased host immune response against tumor cells. Moreover, expression of $\mathrm{T}$ cell-recruiting chemokines positively correlated with improved survival in Ewing sarcoma [25].

\section{Membrane antigens}

The cornerstone of immunotherapy is the ability of the IS to recognize and kill tumor cells. One of the mechanisms is mediated by the encounter between $T$ cells and tumor antigens presented by MHCs on the surface of APCs or on malignant cells themselves. These tumor antigens are peptide epitopes that could theoretically be divided into two main classes: a) non-mutated proteins to which $\mathrm{T}$ cell tolerance is incomplete (for instance, because of their restricted tissue expression pattern); b) peptides not present in the normal human genome, so-called neoantigens. In virus-associated tumors, epitopes derived from viral open reading frames contribute to the pool of neoantigens, while in the larger group of tumors without a viral etiology, such neoantigens are usually generated by tumor-specific DNA mutations that eventually result in the formation of novel proteins [26]. From another point of view, the more a tumor genome is rich in DNA mutations (mutational burden), the greater is the probability that neoantigens are exposed on the surface of tumor cells and the probability of the host IS to generate a response against them.

Unfortunately, this mechanism has two important limitations. The first is the ability of a given antigen to be bound by a MHC class I molecule with a sufficient affin- 
ity to be stabilized on the cell surface in order to activate $\mathrm{T}$ cells by interactions with $\mathrm{T}$ cell receptor (TCR) [27]. The second one, is that high mutational burden has been correlated with response to immunotherapy, but may not be sufficient to cause a relevant clinical benefit, for example, a clinically significant improvement in progression-free survival (PFS). Indeed, data recently presented at the American Society of Clinical Oncology (ASCO) annual meeting from a phase II study of pembrolizumab, in a cohort of patients with advanced soft-tissue or bone sarcoma (SARC028), demonstrated that high mutational burden sarcomas, UPS and LMS, obtained different clinical response rates $(40 \%$ of overall response rate in UPS cohort and $0 \%$ in LMS cohort) suggesting a more complex interaction between mutational burden, TME and IS leading to a greater variability of behavior between different histotypes $[10,28]$.

\section{Metabolic alterations in TME and relationship with IS}

As of today, it is known that cancer cells are characterized by a different metabolism compared to normal cells. These differences also influence TME and IS. In particular, proliferating cancer cells need more energy compared to normal cells, and they adapt their metabolism to be more dependent on aerobic glycolysis and glutaminolysis [29]. Since the metabolic profiles of tumor cells are different from that of normal cells and are critical for their growth and survival, the metabolic signaling pathways have been identified as reasonable targets for therapeutic intervention in patients with cancer.

A well-known example is related to glucose metabolism. Energy within cells is stored by adenosine triphosphate (ATP) molecules. ATP is generated through aerobic or anaerobic respiration. Aerobic respiration, also named oxidative phosphorylation, is used by normal cells under non-proliferating conditions. It produces 36 molecules of ATP per molecule of glucose metabolized, and releases $\mathrm{CO}_{2}$ as a byproduct. Anaerobic respiration, also named anaerobic glycolysis, is used during hypoxic or stressful conditions and produces only two molecules of ATP per molecule of glucose metabolized.

Tumor cells grow rapidly and need to increase the uptake of nutrients from their environment to assemble DNA, proteins, and lipids needed for cell growth and division. In the 1920s, Otto Warburg observed that tumors take up higher levels of glucose in comparison with normal tissues [30, 31]. Furthermore, he showed that cancer cells produce much more lactate than normal tissues, suggesting that these cells are consuming glucose through the glycolytic fermentation pathway [32]. The use of this pathway to meet energy requirements under normoxic conditions is named "aerobic glycolysis", and the increased dependence on this pathway by cancer cells is known as the Warburg effect [33].

In addition to aerobic glycolysis, another critical metabolic pathway to provide nutrients and biosynthetic precursors needed for macromolecule synthesis is the glutamine metabolism [34].

Within this pathway, glutamine is converted into glutamate, used to make alpha-ketoglutarate $(\alpha-K G)$, important in the Krebs cycle for the synthesis of nucleic and fatty acids. Therefore, interfering with glutamine metabolism can have a profoundly detrimental effect on replicating cells [35-38]. The Warburg effect has direct consequences in the TME. The first is the acidification of TME due to lactate secretion and the consequent M2-TAM polarization promoting tumor growth, progression, and metastasis [39]. The second is the reduction of glucose available for TILs with the consequent loss of ability of $\mathrm{T}$ cells to control tumor growth [40]. Thirdly, the Warburg effect is linked to the production of adenosine.

Generation of extracellular adenosine is dependent on the ecto-enzyme CD39 which converts extracellular ATP to AMP, and it depends on the echo-enzyme CD73 which catalyzes the dephosphorylation of AMP to adenosine. Adenosine interacts with four G-protein coupled receptor subtypes with activation of different pathways. In particular, adenosine disables cytotoxic effector functions of both NK and CD8+T cells enabling tumor immune escape. Adenosine inhibits the Th1 CD4+T cell response, thereby limiting the cytokine environment which is needed to support these effector cell types. The polarization of myeloid cells mediated by adenosine allows them to develop into immunosuppressive phenotypes (e.g., M2 macrophages and tolerogenic DCs). Moreover, adenosine enhances proliferation of $\mathrm{T}_{\text {regs }}$ and granulocytic MDSCs which further affect T-effector cells proliferation and function [41-45]. Overexpression of CD73 has been shown in several types of cancer in association with poor prognosis, increased risk of metastasis [46] and resistance to chemotherapy [47, 48]. Preclinical evidence suggests that antibodies targeting CD73 and CD39 have antitumor activity in mouse tumor models [49-53].

Finally, the Warburg effect can confer to tumor cells the ability to resist to hypoxia, a condition very commonly found in solid tumors, wherein tissue oxygenation is generally incomplete due to the inefficient formation of the vascular network [54]. This is particularly relevant in STS that is typically characterized by large masses with large hypoxic areas.

Another critical metabolic pathway is represented by 
indoleamine 2,3-dioxygenase (IDO), that is expressed both by tumor cells and infiltrating myeloid cells, and arginase, produced by MDSCs. In preclinical models, both IDO and arginase have been shown to inhibit immune responses. IDO inhibitors are currently being assessed mainly in combination with immune checkpoint inhibitors in clinical trials involving patients with different cancer types [55], including STS. Interestingly, it has been demonstrated that imatinib activates $\mathrm{CD} 8+\mathrm{T}$ cells and induces the apoptopsis of $\mathrm{T}_{\text {regs }}$ within the tumor by reducing tumor cell expression of IDO [56].

\section{Therapeutic strategies}

\section{Angiogenesis and tumor microenvironment}

Angiogenesis is the formation of new blood vessels by the remodeling and expansion of primary vessels. Tumor angiogenesis is critical for tumor growth and metastasis. To date, different proteins have been identified as angiogenic activators, and it is clear that angiogenesis is a multi-step process. Firstly, hypoxia within the TME determines the activation of hypoxia inducible factor- $1 \alpha$ (HIF-1 $\alpha$ ) that induces the expression of critical angiogenic activators such as vascular endothelial growth factor (VEGF), platelet-derived growth factor (PDGF), and TGF- $\alpha$ [57-59]. Hypoxia also leads to the activation of the signaling of the kinase mammalian target of rapamycin (mTOR) and unfolded protein response (UPR) signaling pathway initiated by stress of the endoplasmic reticulum (ER) [60, 61]. However, different studies have demonstrated that tumor blood vessels are aberrant vessels, different from normal ones [62]. In particular, tumor vessels are deficient in pericytes and have altered endothelium and basement membrane with consequent instability and alteration in blood flow [63]. This aberrant structure reduces the ability of $\mathrm{T}$ lymphocytes to get out of the blood vessels [64]. Preclinical studies are ongoing to evaluate the impact on tumor growth of normalizing the tumor vasculature [65]. Furthermore, other studies are focusing on the combination of antiangiogenic treatments with immunotherapy [64] (Figure 1). Angiogenesis plays a critical role in the pathogenesis of the great majority of tumors, and STS is not an exception $[66,67]$.

\section{Immune stimulation to increase immune infiltrate}

As for other tumors types, sarcoma histotypes can be differentiated into "hot-inflamed" or "cold not-inflamed" tumors and these features, as already mentioned, seem to be an approximate predictive biomarker for response to immunotherapies $[10,28]$. Cold tumors display a completely different immunobiologic signature: lack of type I interferon signature, lack of chemokines for recruitment of $T$ cells, lack of $T$ cells infiltrate, vasculature non-permissive for $\mathrm{T}$ cells entry etc. [68]. Taking into consideration the complete uselessness of mono-immunotherapy in these kind of neoplasms, therapeutic interventions must be focused on a combination of strategies with the aim of increasing inflammatory infiltrate, $T$ cells trafficking and improving innate IS activation.

Possible approaches currently under study are a combination of double checkpoint inhibition (such as the association of anti-CTLA4 and anti-PD1), the use of Toll-like receptor (TLR) agonists and cytokines (such as GM-CSF, IL-2, IFN- $\alpha / \beta)$, vaccines, intratumoral administration of IFN- $\beta$, local radiation therapy, concomitant administration of target therapy or chemotherapy. All of these therapeutic strategies can be called "immune adjuvants", and aim to promote the function of APCs and to enhance Th1 cell or macrophage effector pathways [19]. An attractive example is the study published by Chen and collaborators, in which the combination of peginterferon $\alpha-2 b$ and imatinib for treatment of patients with stage III/IV GIST promoted DCs and cytotoxic T lymphocytes differentiation toward Th1 response and activation of NK cells with promising clinical responses (albeit the number of treated patients was small) [69]. Another interesting combination therapy is represented by ipilimumab + nivolumab, that has been studied in the phase II study Alliance A091401, recently reported at the 2017 ASCO annual meeting. Patients with advanced sarcoma, progressing after at least one treatment line, received nivolumab $3 \mathrm{mg} / \mathrm{kg}$ alone or in combination with ipilimumab $1 \mathrm{mg} / \mathrm{kg}$. The combination treatment group obtained two complete and five partial responses (objective response rate [ORR] 15\%), primarily in LMS, UPS, myxofibrosarcoma, and angiosarcoma, compared to only three partial responses in the monotherapy group (ORR $6 \%$ ); PFS was slightly greater in combination treatment (PFS 4.4 vs 2.1 months) justifying further studies of this combination in selected patient populations [70].

\section{Toll-like receptors}

Toll like receptors (TLRs) are the best-known representatives of the molecules of the pattern recognition receptor (PRR) family. They are expressed by cells of innate immunity, DCs and macrophages, but also by epithelial cells and fibroblasts. They can recognize "non-self", albeit in a less specific way than cells of adaptive immunity. In fact, TLRs detect specific molecular signatures, "patterns" commonly present on pathogens. The human genome harbors 10 TLR genes; each of these can recog- 


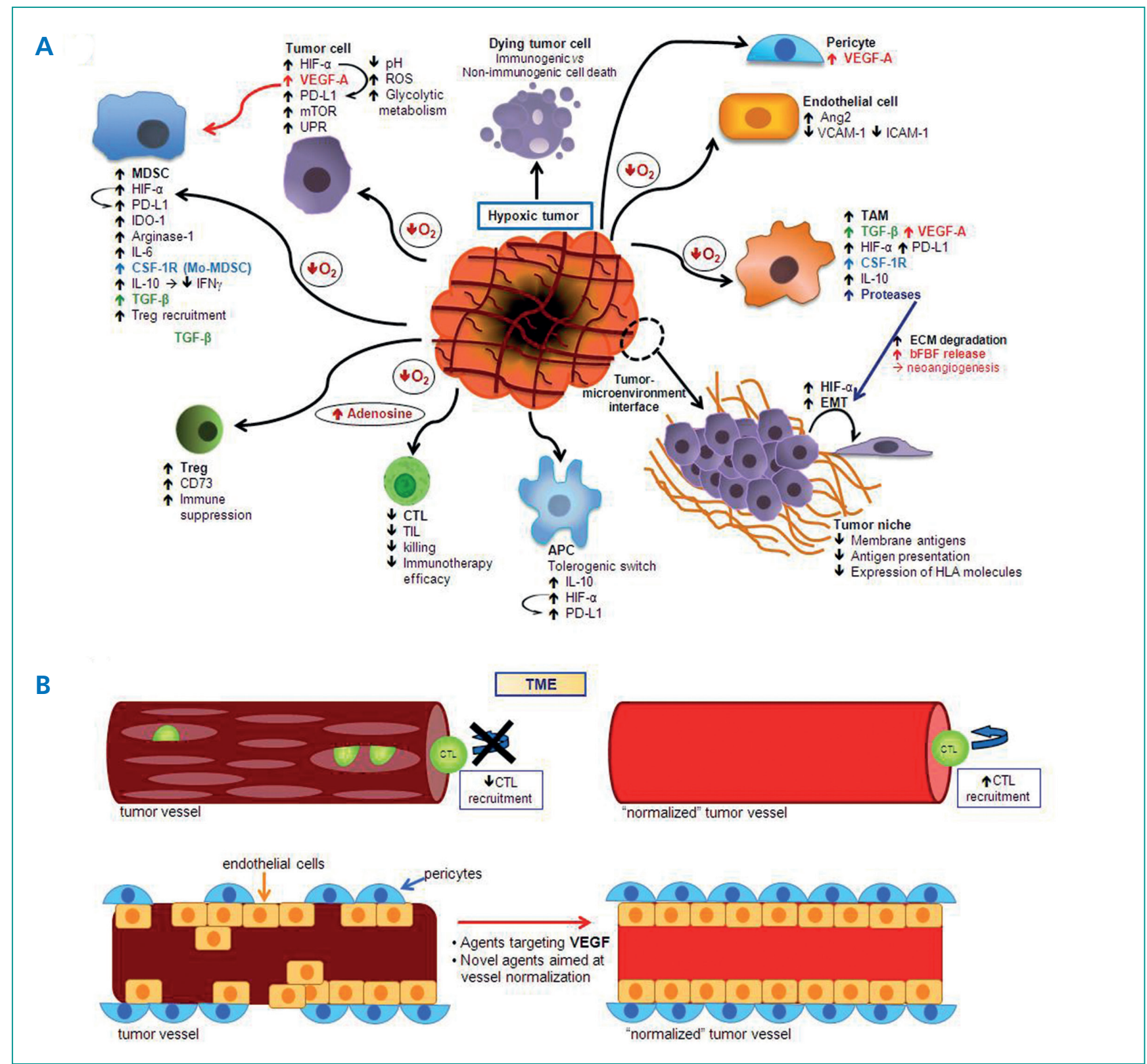

Fig. 1. Interactions between tumor hypoxia and the immune system: a complex interplay. A. Growing, hypoxic tumor masses producing hypoxia-inducible factor $\alpha(\mathrm{HIF}-\alpha)$ stimulate the production of angiogenic factors by tumor cells, (i.e., members of the vascular endothelial growth factor-VEGF family), and by endothelial cells, (i.e., angiopoietin 2 - Ang2). Moreover, hypoxia can also induce epithelial-mesenchymal transitions (EMT) in cancer cells. Tumor neoangiogenesis gives rise to heterogeneous, leaky and tortuous vessels, more susceptible to cancer cell intravasation. The proangiogenic molecules activate signaling pathways leading to different effects: i) tumor immunosuppression; ii) reduced trafficking of cytotoxic T lymphocytes (CTL) by inducing the so-called "endothelial anergy" (inhibition of adhesion molecules usually increased in inflammatory states); iii) increased extravasation of $\mathrm{T}_{\text {regs }}$; iv) recruitment of MDSC with increased activity of various MDSC-associated cytokines, including indoleamine 2,3-dioxygenase 1 (IDO1) and arginase-1, both involved in antitumor immune response inhibition. Hypoxia is a well-known attractor of tumor-associated macrophages (TAM) that produce proangiogenic cytokines and other tumor-promoting cytokines. Other pathways activated by hypoxic conditions in tumor cells are the mammalian target of rapamycin (mTOR) and unfolded protein response (UPR) pathways, that can contribute to tumor progression and modulate tumor tolerance to hypoxia. Furthermore, hypoxia directly upregulates PD-L1 in cancer cells, TAM, and MDSC (HIF- $\alpha$ binds to a hypoxia-response element in the PD-L1 promoter).

B. Upon hypoxic conditions, the phenotypic switch of endothelial cells and pericytes leads to neoangiogenesis. In tumor vessels, endothelial cells lose their typical "phalanx-like" asset, with detachment from the basement membrane, loss of cell polarity, increase in fenestration number and pore size, generating a leaky, heterogeneous endothelial layer more permissive for intravasation of tumor cells. However, quite counterintuitively, this does not lead to increased extravasation of CTL in the TME, as the phenotypic switch turns endothelial cells into cells hostile to CTL migration. On the other hand, the same phenotypic switch leads to a more favorable environment for $\mathrm{T}_{\mathrm{reg}}$ extravasation. Pericytes of tumor vessels, the "guardians" of vessel walls, usually become fewer and display an overall less mature phenotype. Therefore, the use of antiangiogenic agents and novel agents aimed at vessel normalization (i.e., molecules stimulating Notch signaling pathway) could prove to be a powerful tool in order to boost the efficacy of immunotherapy. APC: antigen presenting cell; bFBF: basic fibroblast growth factor; ECM: extracellular matrix; ICAM-1: intercellular adhesion molecule-1; Mo-MDSC: monocytic myeloid derived suppressor cells; TIL: tumor infiltrating lymphocytes; TME: tumor microenvironment; VCAM-1: vascular cell adhesion molecule-1. 
nize a specific molecular class, such as proteins, lipoproteins, proteins, nucleic acids, and glycans. Those recognizing nucleic acids (bacterial, viral, and self in autoimmunity) are not expressed on the cell surface, but in the intracellular compartment (ER/endosomes/lysosomes). TLRs can also recognize endogenous molecules released from apoptotic/necrotizing cancer cells, and TLRs gene mutations are linked to cancer development and progression. Indeed, it is quite likely that the antitumor effect observed by Coley in 1890 on injection of streptococcal toxin might have been mediated by TLR, specifically by TLR4, which recognizes lipopolysaccharide (LPS) [71]. TLR agonists have proven to be a promising target to enhance antitumor immune response. The rationale behind this intuition relies on the role of these molecules in antigen presentation. One of the first, seminal studies in this direction was the discovery in 2005 of the reversal of $\mathrm{T}_{\text {regs }}$ function (from negative to positive regulators of antitumor immunity) by adoptive transfer of TLR8 ligand-stimulated $\mathrm{T}_{\text {regs }}$ into tumor-bearing mice [72]. The availability of precise structural knowledge of TLRs ligand binding domains since 2005 has allowed the development of highly structure-specific agonists [73, 74]. To date, there are a number of TLR agonists, such as imiquimod (activating TLR7) or CpG oligonucleotides (activating TLR9). Different TLRs have specific effects on antitumor immune response. For instance, while TLR2-4-7 have a tumor-promoting effect, TLR9 activation could promote immune responses against cancer cells. In order to tackle this "Janus effect" across the TLR family, more basic molecular biology details are needed to identify which adjuvant strategies and which TLRs might be particularly helpful as antitumor immunity enhancers [19]. One recent promising study in this direction combined radiation therapy with the use of a powerful TLR4 agonist, the lipid adjuvant G100 (oil-in-water stable glucopyranosyl emulsion), for intratumoral injections, in order to try and induce an immune response in the TME. The study was carried out in a small number of patients affected by metastatic STS, with promising results [75].

\section{Strategies to improve antigen presentation}

While, on the one hand, we can improve IS sensitivity with newer immune adjuvants, on the other hand, preclinical studies have shown that "classical" therapies (such as chemotherapy, radiotherapy and target therapy) should not be discarded. Indeed, it is reasonable to combine standard therapies with immunotherapies to increase antigen release by the dying cancer cell [76].

Radiotherapy, aside from its tumor-debulking properties, modulates the antitumor immune response in a variety of ways, such as the release of tumor antigens and/or damage-associated molecular pattern molecules (DAMPs), that can activate both the innate and adaptive IS. Indeed, it can improve tumor-cell immunogenicity, enhancing APC function and T cell effector activity [77]. Furthermore, the primary effects of radiotherapy are on the tissues and target lesions, but the activation of IS can also lead to activity against distant lesions (the so-called "abscopal effect"). The abscopal effect is not completely understood, but it can be considered an indirect proof of benefit and synergistic action of radiotherapy and IS. Similarly, chemotherapy can also induce immunogenic tumor cell death (ICD). The main molecular "actors" released by tumor cells after the administration of chemotherapy (e.g., anthracycline and oxaliplatin) are: 1) calreticulin, which favors antigen-uptake; 2) ATP, which represents a potent chemotactic agent for myeloid cells and DCs precursors; 3) high mobility group box-1 (HMGB-1), that favors antigen presentation by DCs [76].

Targeted therapies have been shown to modulate the interaction between tumor and IS even if the mechanisms are not yet completely understood. Once again, studies in melanoma paved the way to discoveries in other tumor types. BRAF inhibitors and the combination of BRAF and MEK inhibitors reduce immune suppression within the TME through mechanisms such as the increase in the expression of melanocyte differentiation antigens and stimulation of lymphocyte homing [78]. As mentioned above, one of the most attractive examples in tumors other than melanoma is the block of IDO-mediated immunosuppression of $\mathrm{T}$ cell responses by imatinib in GIST. In particular, this drug causes the reduction of the expression of IDO within the tumor, causing $\mathrm{T}_{\text {regs }}$ apoptosis and activation of CD8+T cells [56].

\section{Conclusions}

The interactions between cancer, IS and TME appear to be very complex and are far from having been completely elucidated. Complexity is much greater than that hypothesized by Coley when he observed responses to his "toxin", but also clearly much more complex than expected by oncologists all over the world after the treatment of melanoma patients with checkpoint inhibitors. The unmet need is twofold. First, we should not be satisfied with the results, albeit practice changing, obtained in melanoma, NSCLC, RCC, Merkel cell carcinoma (MCC), and urothelial carcinoma revolutionizing the treatment algorithm of these tumors. A proportion of patients affected by these same tumors die or lose their initial response in a short time. Second, in many other tumors, from colorectal cancer (apart from MSH related ones) to rare cancers such as sarcomas, present im- 
Table 1. Examples of strategies to modulate the tumor microenvironment (TME).

\begin{tabular}{|c|c|c|}
\hline TME modulators & Potential therapeutic strategies & References \\
\hline Antigen load - neoantigens & $\begin{array}{l}\text { - Vaccines targeting NY-ESO-1; dendritic-cell-based vaccines; vaccines composed } \\
\text { of multiple tumor associated peptides (TUMAPs) } \\
\text { - Oncolytic viruses: talimogene laherparepvec, an attenuated herpes simplex virus } \\
\text { type } 1 \text { (HSV-1); pexastimogene devacirepvec, based on an oncolytic vaccinia poxvir } \\
\text { modified human reovirus; recombinant adenovirus } \\
\text { - Combination with radiotherapy and chemotherapy }\end{array}$ & {$[26,79-86]$} \\
\hline Immune checkpoints & - Immune checkpoint inhibitors targeting CTLA-4, PD-1, PD-L1 & \\
\hline Antigen presentation adjuvants & $\begin{array}{l}\text { - TLR agonists/adjuvants: ARNAX (TLR3-specific RNA agonist), imiquimod, } \\
\alpha \text {-Galactosylceramide }\end{array}$ & {$[87,88]$} \\
\hline Tumor infiltrating lymphocytes & $\begin{array}{l}\text { - Adoptive cell transfer } \\
\text { - CAR-T } \\
\text { - Cytokines agonists/antagonists (see below) } \\
\text { - Double checkpoint inhibition } \\
\text { - Target therapy }\end{array}$ & {$[78,89-91]$} \\
\hline Tumor associated macrophages & - CSF1-R inhibitors/anti-CSF1-R antibodies; anti-CD47 antibodies & [92] \\
\hline Tumor metabolism & $\begin{array}{l}\text { - IDO inhibitors } \\
\text { - Arginase inhibitors } \\
\text { - Anti-CD73 antibodies } \\
\text { - Anti-CD39 antibodies } \\
\text { - A2B adenosine receptor blockers (PSB603) }\end{array}$ & $\begin{array}{r}{[49,50,52} \\
53,55]\end{array}$ \\
\hline Tumor neoangiogenesis & $\begin{array}{l}\text { - VEGF inhibition } \\
\text { - HDAC, DNMT inhibition }\end{array}$ & {$[57,91-94]$} \\
\hline $\begin{array}{l}\text { Cytokines, chemokines, } \\
\text { cytokine receptors }\end{array}$ & - Cytokines agonists/antagonists: IL-1 $\alpha$, IL-2, CCL2, Interferons, TGF- $\beta[\ldots]$ & {$[20,95-100]$} \\
\hline
\end{tabular}

CAR-T: chimeric antigen receptor T cells; DNMT: DNA methyltransferase; HDAC: histone deacetylases; IDO: indoleamine-2,3-dioxygenase

munotherapy strategies have not shown any relevant activity. We need to translate into clinical trials what we have learned from preclinical studies so as to approach the treatment of "non-melanoma-like cancers" with a multitasking ability. Thus, the effort has to be focused on the modulation of the properties of the immune system and on the combination of immunotherapies with standard therapies and/or other therapeutic strategies, such as adoptive cell therapies and modulation of cancer metabolism (Table 1).

\section{Acknowledgments}

The Authors thank Ray Hill, an independent medical writer, who provided native English editing and journal styling on behalf of HPS. This editorial assistance was funded by PharmaMar, Spain.

\section{Conflicts of Interest}

The Authors declare there are no conflicts of interest in relation to this article. 


\section{References}

1. Coley WB. The treatment of malignant tumours by repeated inoculations of erysipelas with a report of ten original cases. Am J Med Sci 1893;105:487-511.

2. Parish CR. Cancer immunotherapy: the past, the present and the future. Immunol Cell Biol 2003;81(2):106-13.

3. Sharma P, Allison JP. The future of immune checkpoint therapy. Science 2015;348(6230):56-61.

4. D'Angelo SP, Shoushtari AN, Keohan ML et al. Combined KIT and CTLA-4 blockade in patients with refractory GIST and other advanced sarcomas: a phase Ib study of dasatinib Plus ipilimumab. Clin Cancer Res 2017;23(12):2972-80.

5. Teng MW, Ngiow SF, Ribas A et al. Classifying cancers based on T-cell infiltration and PD-L1. Cancer Res 2015;75(11):2139-45.

6. Taube JM, Klein A, Brahmer JR et al. Association of PD-1, PD-1 ligands, and other features of the tumor immune microenvironment with response to anti-PD-1 therapy. Clin Cancer Res 2014;20(19):5064-74.

7. Tumeh PC, Harview CL, Yearley JH et al. PD-1 blockade induces responses by inhibiting adaptive immune resistance. Nature 2014;515(7528):568-71.

8. Fletcher CDM, Bridge JA, Hogendoorn P, Mertens F. WHO Classification of Tumours of Soft Tissue and Bone. 4th ed. WHO Lyon: IARC Press; 2013.

9. Fletcher CD. The evolving classification of soft tissue tumours-an update based on the new 2013 WHO classification. Histopathology 2014;64(1):2-11.

10. Pollack SM, He Q, Yearley JH et al. T-cell infiltration and clonality correlate with programmed cell death protein 1 and programmed death-ligand 1 expression in patients with soft tissue sarcomas. Cancer 2017; doi: 10.1002/cncr.30726. [Epub ahead of print].

11. George S, Barysauskas CM, Solomon S et al. Phase 2 study of nivolumab in metastatic leiomyosarcoma of the uterus. J Clin Oncol 2016;34(15_suppl):11007.

12. Polyak K, Weinberg RA. Transitions between epithelial and mesenchymal states: acquisition of malignant and stem cell traits. Nat Rev Cancer 2009;9(4):265-73.

13. Zavadil J, Bottinger EP. TGF-beta and epithelial-to-mesenchymal transitions. Oncogene 2005;24(37):5764-74.

14. Blobe GC, Schiemann WP, Lodish HF. Role of transforming growth factor $\beta$ in human disease. N Engl J Med 2000;342(18):1350-8.

15. Elliott RL, Blobe GC. Role of transforming growth factor Beta in human cancer. J Clin Oncol 2005;23(9):2078-93.

16. Massague J, Gomis RR. The logic of TGFbeta signaling. FEBS Lett 2006;580(12):2811-20.

17. Anzano MA, Roberts AB, Smith JM et al. Sarcoma growth factor from conditioned medium of virally transformed cells is composed of both type alpha and type beta transforming growth factors. Proc Natl Acad Sci U S A 1983; 80(20):6264-8.

18. Hahm KB, Cho K, Lee C et al. Repression of the gene encoding the TGF-beta type II receptor is a major target of the EWS-FLI1 oncoprotein. Nat Genet 1999;23(2):222-7.

19. Smyth MJ, Ngiow SF, Ribas A et al. Combination cancer immunotherapies tailored to the tumour microenvironment. Nat Rev Clin Oncol 2016;13(3):143-58.
20. Lippitz BE. Cytokine patterns in patients with cancer: a systematic review. Lancet Oncol 2013;14(6):e218-e28.

21. Saraiva M, O'Garra A. The regulation of IL-10 production by immune cells. Nat Rev Immunol 2010;10(3):170-81.

22. Shouval DS, Ouahed J, Biswas A et al. Interleukin 10 receptor signaling: Master regulator of intestinal mucosal homeostasis in mice and humans. Adv Immunol 2014; 122:177-210.

23. Ruffell B, Chang-Strachan D, Chan V et al. Macrophage IL-10 blocks CD8+ T cell-dependent responses to chemotherapy by suppressing IL-12 expression in intratumoral dendritic cells. Cancer Cell 2014;26(5):623-37.

24. Pyonteck SM, Akkari L, Schuhmacher AJ et al. CSF-1R inhibition alters macrophage polarization and blocks glioma progression. Nat Med 2013;19(10):1264-72.

25. Berghuis D, Santos SJ, Baelde HJ et al. Pro-inflammatory chemokine-chemokine receptor interactions within the Ewing sarcoma microenvironment determine CD8+ T-lymphocyte infiltration and affect tumour progression. J Pathol 2011;223(3):347-57.

26. Schumacher TN, Schreiber RD. Neoantigens in cancer immunotherapy. Science 2015;348(6230):69-74.

27. Yewdell JW, Bennink JR. Immunodominance in major histocompatibility complex class I-restricted T lymphocyte responses. Annu Rev Immunol 1999;17(1):51-88.

28. Burgess MA, Bolejack V, Van Tine BA et al. Multicenter phase II study of pembrolizumab (P) in advanced soft tissue (STS) and bone sarcomas (BS): Final results of SARC028 and biomarker analyses [Abstract]. J Clin Oncol 2017;35(Suppl.):abstr 11008.

29. Weyandt JD, Thompson CB, Giaccia AJ et al. Metabolic alterations in cancer and their potential as therapeutic targets. American Society of Clinical Oncology Educational Book 2017;63:8955-61.

30. Warburg O. Stoffwechsel der Karzinomzelle. Verhandlungen der Deutschen Gesellschaft für Innere Medizin. Springer; 1928. p. 11-8.

31. Warburg O. Ueber den Stoffwechsel der Tumoren (The metabolism of tumours; investigations from the Kaiser Wilhelm institute for biology, Berlin-Dahlem. J Am Med Assoc 1931;96:1982-2309.

32. Warburg O. On the origin of cancer. Science 1956;123(3191): 309-14.

33. Dang CV. Links between metabolism and cancer. Genes Dev 2012;26(9):877-90.

34. DeBerardinis RJ, Chandel NS. Fundamentals of cancer metabolism. Sci Adv 2016;2(5):e1600200.

35. Xiang Y, Stine ZE, Xia J et al. Targeted inhibition of tumorspecific glutaminase diminishes cell-autonomous tumorigenesis. J Clin Invest 2015;125(6):2293-306.

36. Momcilovic M, Bailey ST, Lee JT et al. Targeted inhibition of EGFR and glutaminase induces metabolic crisis in EGFR mutant lung cancer. Cell Reports 2017;18(3): 601-10.

37. Gross MI, Demo SD, Dennison JB et al. Antitumor activity of the glutaminase inhibitor CB-839 in triple-negative breast cancer. Mol Cancer Ther 2014;13(4):890-901.

38. Wang J-B, Erickson JW, Fuji R et al. Targeting mitochon- 
drial glutaminase activity inhibits oncogenic transformation. Cancer Cell 2010;18(3):207-19.

39. Colegio OR, Chu N-Q, Szabo AL et al. Functional polarization of tumour-associated macrophages by tumour-derived lactic acid. Nature 2014;513(7519):559-63.

40. Chang C-H, Qiu J, O’Sullivan D et al. Metabolic competition in the tumor microenvironment is a driver of cancer progression. Cell 2015;162(6):1229-41.

41. Stagg J, Smyth M. Extracellular adenosine triphosphate and adenosine in cancer. Oncogene 2010;29(39):5346-58.

42. Deaglio S, Dwyer KM, Gao W et al. Adenosine generation catalyzed by CD39 and CD73 expressed on regulatory $\mathrm{T}$ cells mediates immune suppression. $\mathrm{J}$ Exp Med 2007;204(6):1257-65.

43. Novitskiy SV, Ryzhov S, Zaynagetdinov R et al. Adenosine receptors in regulation of dendritic cell differentiation and function. Blood 2008;112(5):1822-31.

44. Ryzhov S, Novitskiy SV, Goldstein AE et al. Adenosinergic regulation of the expansion and immunosuppressive activity of CD11b+ Gr1+ cells. J Immunol 2011;187(11): 6120-9.

45. Ohta A, Kini R, Ohta A et al. The development and immunosuppressive functions of CD4(+) CD25(+) FoxP3(+) regulatory $\mathrm{T}$ cells are under influence of the adenosine-A2A adenosine receptor pathway. Front Immunol 2012;3:190.

46. Leth-Larsen R, Lund R, Hansen HV et al. Metastasis-related plasma membrane proteins of human breast cancer cells identified by comparative quantitative mass spectrometry. Mol Cell Proteomics 2009;8(6):1436-49.

47. Serra S, Horenstein AL, Vaisitti T et al. CD73-generated extracellular adenosine in chronic lymphocytic leukemia creates local conditions counteracting drug-induced cell death. Blood 2011;118(23):6141-52.

48. Loi S, Pommey S, Haibe-Kains B et al. CD73 promotes anthracycline resistance and poor prognosis in triple negative breast cancer. Proc Natl Acad Sci U S A 2013;110(27):11091-6.

49. Stagg J, Divisekera U, Duret $\mathrm{H}$ et al. CD73-deficient mice have increased antitumor immunity and are resistant to experimental metastasis. Cancer Res 2011;71(8):2892-900.

50. Sun X, Wu Y, Gao W et al. CD39/ENTPD1 expression by $\mathrm{CD} 4+$ Foxp3+ regulatory $\mathrm{T}$ cells promotes hepatic metastatic tumor growth in mice. Gastroenterology 2010; 139(3):1030-40.

51. Wang L, Fan J, Thompson LF et al. CD73 has distinct roles in nonhematopoietic and hematopoietic cells to promote tumor growth in mice. J Clin Invest 2011;121(6):2371-82.

52. Mediavilla-Varela M, Luddy K, Noyes D et al. Antagonism of adenosine A2A receptor expressed by lung adenocarcinoma tumor cells and cancer associated fibroblasts inhibits their growth. Cancer Biol Ther 2013;14(9):860-8.

53. Wei Q, Costanzi S, Balasubramanian R et al. A2B adenosine receptor blockade inhibits growth of prostate cancer cells. Purinergic Signal 2013;9(2):271-80.

54. Pelicano H, Martin D, Xu R et al. Glycolysis inhibition for anticancer treatment. Oncogene 2006;25(34):4633-46.

55. Munn DH. Blocking IDO activity to enhance anti-tumor immunity. Front Biosci 2011;4:734-45.

56. Balachandran VP, Cavnar MJ, Zeng S et al. Imatinib potentiates antitumor $\mathrm{T}$ cell responses in gastrointestinal stromal tumor through the inhibition of Ido. Nat Med 2011;17(9):1094-100.

57. Fukumura D, Jain RK. Tumor microvasculature and microenvironment: targets for anti-angiogenesis and normalization. Microvasc Res 2007;74(2):72-84.

58. Melillo G. Inhibiting hypoxia-inducible factor 1 for cancer therapy. Mol Cancer Res 2006;4(9):601-5.

59. Kaur B, Khwaja FW, Severson EA et al. Hypoxia and the hypoxia-inducible-factor pathway in glioma growth and angiogenesis. Neuro Oncol 2005;7(2):134-53.

60. Wouters BG, Koritzinsky M. Hypoxia signalling through $\mathrm{mTOR}$ and the unfolded protein response in cancer. Nat Rev Cancer 2008;8(11):851.

61. Land SC, Tee AR. Hypoxia-inducible factor $1 \alpha$ is regulated by the mammalian target of rapamycin (mTOR) via an mTOR signaling motif. J Biol Chem 2007;282(28):20534-43.

62. Konerding M, Malkusch W, Klapthor B et al. Evidence for characteristic vascular patterns in solid tumours: quantitative studies using corrosion casts. Br J Cancer 1999;80 (5-6):724.

63. Baluk P, Hashizume H, McDonald DM. Cellular abnormalities of blood vessels as targets in cancer. Curr Opin Genet Dev 2005;15(1):102-11.

64. Schmittnaegel M, Rigamonti N, Kadioglu E et al. Dual angiopoietin-2 and VEGFA inhibition elicits antitumor immunity that is enhanced by PD- 1 checkpoint blockade. Sci Transl Med 2017;9(385):eaak9670.

65. Jain RK. Normalization of tumor vasculature: an emerging concept in antiangiogenic therapy. Science 2005; 307(5706):58-62.

66. Sleijfer S, van der Graaf WT, Blay J-Y. Angiogenesis inhibition in non-GIST soft tissue sarcomas. Oncologist 2008;13(11):1193-200.

67. Judson I, Verweij J, Gelderblom H et al. Doxorubicin alone versus intensified doxorubicin plus ifosfamide for firstline treatment of advanced or metastatic soft-tissue sarcoma: a randomised controlled phase 3 trial. Lancet Oncol 2014;15(4):415-23.

68. Gajewski TF, Schreiber H, Fu Y-X. Innate and adaptive immune cells in the tumor microenvironment. Nat Immunol 2013;14(10):1014-22.

69. Chen LL, Chen X, Choi H et al. Exploiting antitumor immunity to overcome relapse and improve remission duration. Cancer Immunol Immunother 2012;61(7):1113-24.

70. D’Angelo SP, Mahoney MR, Van Tine BA et al. A multi-center phase II study of nivolumab+/-ipilimumab for patients with metastatic sarcoma (Alliance A091401) [Abstract]. J Clin Oncol 2017;35(Suppl):abstr 11007.

71. Rakoff-Nahoum S, Medzhitov R. Toll-like receptors and cancer. Nat Rev Cancer 2009;9(1):57-63.

72. Peng G, Guo Z, Kiniwa Y et al. Toll-like receptor 8-mediated reversal of CD4+ regulatory $\mathrm{T}$ cell function. Science 2005;309(5739):1380-4.

73. Choe J, Kelker MS, Wilson IA. Crystal structure of human toll-like receptor 3 (TLR3) ectodomain. Science 2005;309(5734):581-5.

74. Bell JK, Botos I, Hall PR et al. The molecular structure of the Toll-like receptor 3 ligand-binding domain. Proc Natl Acad Sci U S A 2005;102(31):10976-80. 
75. Seo YD, Kim EY, Conrad EU et al. Intratumoral injection of the toll-like receptor 4 agonist G100 induces a T-cell response in the soft tissue sarcoma microenvironment [Abstract]. Cancer Res 2017;77(13 Suppl):abstr 2947.

76. Zitvogel L, Galluzzi L, Smyth MJ et al. Mechanism of action of conventional and targeted anticancer therapies: reinstating immunosurveillance. Immunity 2013;39(1):74-88.

77. Demaria S, Formenti SC. Radiation as an immunological adjuvant: current evidence on dose and fractionation. Front Oncol 2012;2:153.

78. Hu-Lieskovan S, Robert L, Homet Moreno B et al. Combining targeted therapy with immunotherapy in BRAFmutant melanoma: promise and challenges. J Clin Oncol 2014;32(21):2248-54.

79. Lichty BD, Breitbach CJ, Stojdl DF et al. Going viral with cancer immunotherapy. Nat Rev Cancer 2014;14(8):559-67.

80. Rosenberg SA, Restifo NP. Adoptive cell transfer as personalized immunotherapy for human cancer. Science 2015;348(6230):62-8.

81. Robbins PF, Morgan RA, Feldman SA et al. Tumor regression in patients with metastatic synovial cell sarcoma and melanoma using genetically engineered lymphocytes reactive with NY-ESO-1. J Clin Oncol 2011;29(7):917-24.

82. Krishnadas DK, Shusterman S, Bai F et al. A phase I trial combining decitabine/dendritic cell vaccine targeting MAGE-A1, MAGE-A3 and NY-ESO-1 for children with relapsed or therapy-refractory neuroblastoma and sarcoma. Cancer Immunol Immunother 2015;64(10):1251-60.

83. Dhodapkar MV, Sznol M, Zhao B et al. Induction of antigen-specific immunity with a vaccine targeting NY-ESO-1 to the dendritic cell receptor DEC-205. Sci Transl Med 2014;6(232):232ra51.

84. Ngiow SF, McArthur GA, Smyth MJ. Radiotherapy complements immune checkpoint blockade. Cancer Cell 2015;27(4):437-8.

85. Palucka K, Banchereau J. Dendritic-cell-based therapeutic cancer vaccines. Immunity 2013;39(1):38-48.

86. Walter S, Weinschenk T, Stenzl A et al. Multipeptide immune response to cancer vaccine IMA901 after single-dose cyclophosphamide associates with longer patient survival. Nat Med 2012;18(8):1254-61.

87. Gableh F, Saeidi M, Hemati S et al. Combination of the toll like receptor agonist and $\alpha$-Galactosylceramide as an efficient adjuvant for cancer vaccine. J Biomed Sci 2016;23(1):16.

88. Takeda Y, Kataoka K, Yamagishi J et al. A TLR3-specific adjuvant relieves innate resistance to PD-L1 blockade with- out cytokine toxicity in tumor vaccine immunotherapy. Cell Rep 2017;19(9):1874-87.

89. Goff SL, Dudley ME, Citrin DE et al. Randomized, prospective evaluation comparing intensity of lymphodepletion before adoptive transfer of tumor-infiltrating lymphocytes for patients with metastatic melanoma. J Clin Oncol 2016;34(20):2389-97.

90. Maude SL, Frey N, Shaw PA et al. Chimeric antigen receptor T cells for sustained remissions in leukemia. N Engl J Med 2014;371(16):1507-17.

91. Vanneman M, Dranoff G. Combining immunotherapy and targeted therapies in cancer treatment. Nat Rev Cancer 2012;12(4):237-51.

92. Mantovani A, Marchesi F, Malesci A et al. Tumour-associated macrophages as treatment targets in oncology. Nat Rev Clin Oncol 2017;14(7):399-416.

93. Braghiroli MI, Sabbaga J, Hoff PM. Bevacizumab: overview of the literature. Expert Rev Anticancer Ther 2012;12(5):567-80.

94. Falkenberg KJ, Johnstone RW. Histone deacetylases and their inhibitors in cancer, neurological diseases and immune disorders. Nat Rev Drug Discov 2014;13(9):673-91.

95. Linch M, Miah AB, Thway K et al. Systemic treatment of soft-tissue sarcoma-gold standard and novel therapies. Nat Rev Clin Oncol 2014;11(4):187-202.

96. McDermott D, Ghebremichael M, Signoretti S et al. The high-dose aldesleukin (HD IL-2) "SELECT" trial in patients with metastatic renal cell carcinoma (mRCC). J Clin Oncol 2010;28(15_suppl):4514.

97. Pienta KJ, Machiels JP, Schrijvers D et al. Phase 2 study of carlumab (CNTO 888), a human monoclonal antibody against CC-chemokine ligand 2 (CCL2), in metastatic castration-resistant prostate cancer. Invest New Drugs 2013;31(3):760-8.

98. Hong DS, Janku F, Naing A et al. Xilonix, a novel true human antibody targeting the inflammatory cytokine interleukin-1 alpha, in non-small cell lung cancer. Invest New Drugs 2015;33(3):621-31.

99. Lewis AM, Varghese $\mathrm{S}, \mathrm{Xu} \mathrm{H}$ et al. Interleukin-1 and cancer progression: the emerging role of interleukin-1 receptor antagonist as a novel therapeutic agent in cancer treatment. J Trans1 Med 2006;4(1):48.

100. Rodon J, Carducci MA, Sepulveda-Sanchez JM et al. Firstin-human dose study of the novel transforming growth factor-beta receptor I kinase inhibitor LY2157299 monohydrate in patients with advanced cancer and glioma. Clin Cancer Res 2015;21(3):553-60. 